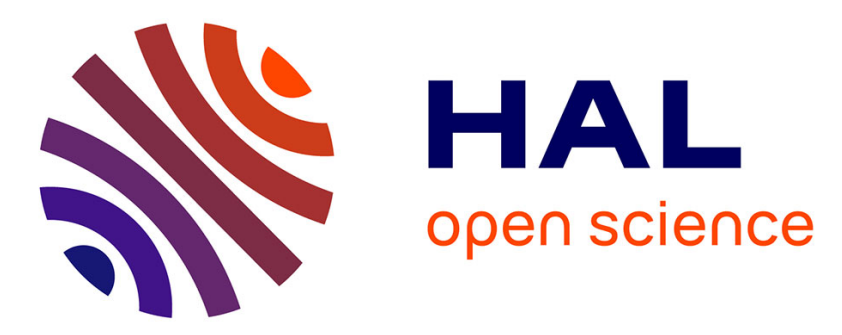

\title{
Numerical study of a Flexible Sail Plan submitted to pitching: Hysteresis phenomenon and effect of rig Adjustments
}

Benoit Augier, Frédéric Hauville, Patrick Bot, Nicolas Aubin, Mathieu Durand

\section{- To cite this version:}

Benoit Augier, Frédéric Hauville, Patrick Bot, Nicolas Aubin, Mathieu Durand. Numerical study of a Flexible Sail Plan submitted to pitching: Hysteresis phenomenon and effect of rig Adjustments. 2014. hal-01071526

\author{
HAL Id: hal-01071526 \\ https://hal.science/hal-01071526
}

Preprint submitted on 8 Oct 2014

HAL is a multi-disciplinary open access archive for the deposit and dissemination of scientific research documents, whether they are published or not. The documents may come from teaching and research institutions in France or abroad, or from public or private research centers.
L'archive ouverte pluridisciplinaire HAL, est destinée au dépôt et à la diffusion de documents scientifiques de niveau recherche, publiés ou non, émanant des établissements d'enseignement et de recherche français ou étrangers, des laboratoires publics ou privés. 


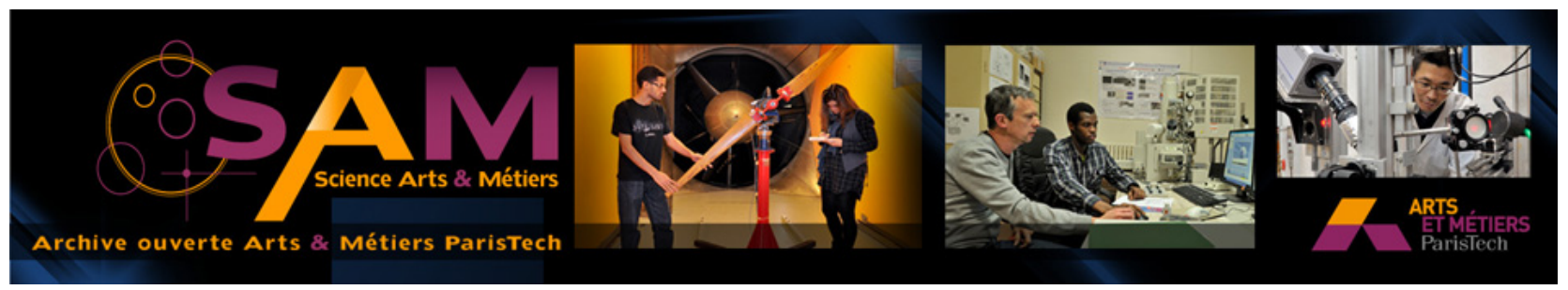

Science Arts \& Métiers (SAM)

is an open access repository that collects the work of Arts et Métiers ParisTech researchers and makes it freely available over the web where possible.

This is an author-deposited version published in: http://sam.ensam.eu

Handle ID: .http://hdl.handle.net/10985/8688

\section{To cite this version :}

Benoit AUGIER, Frédéric HAUVILLE, Patrick BOT, Nicolas AUBIN, Mathieu DURAND Numerical study of a Flexible Sail Plan submitted to pitching: Hysteresis phenomenon and effect of rig Adjustments - Ocean Engineering p.à paraitre - 2014 


\title{
Numerical study of a Flexible Sail Plan submitted to pitching: Hysteresis phenomenon and effect of rig Adjustments
}

\author{
Benoit Augier ${ }^{\curvearrowright}$, Frederic Hauville ${ }^{\curvearrowright}$, Patrick Bot $^{\curvearrowright}$, Nicolas Aubin ${ }^{\curvearrowright}$, Mathieu \\ Durand $^{\Upsilon \text { a }}$ \\ ${ }^{9}$ Naval Academy Research Institute - IRENAV CC600, 29240 BREST Cedex 9, France \\ ^K-Epsilon Company, 1300 route des Cretes - B.P 25506905 Sophia Antipolis Cedex, France
}

\begin{abstract}
A numerical investigation of the dynamic Fluid Structure Interaction (FSI) of a yacht sail plan submitted to harmonic pitching is presented to analyse the system's dynamic behaviour and the effects of motion simplifications and rigging adjustments on aerodynamic forces. It is shown that the dynamic behaviour of a sail plan subject to yacht motion clearly deviates from the quasi-steady theory. The aerodynamic forces presented as a function of the instantaneous apparent wind angle show hysteresis loops. It is shown that the hysteresis phenomenon dissipates some energy and that the dissipated energy increases strongly with the pitching reduced frequency and amplitude. The effect of reducing the real pitching motion to a simpler surge motion is investigated. Results show significant discrepancies with underestimated aerodynamic forces and no more hysteresis when a surge motion is considered. However, the superposition assumption consisting in a decomposition of the surge into two translations normal and collinear to the apparent wind is
\end{abstract}

\footnotetext{
Email address: patrick.bot@ecole-navale.fr Tel:+33 298233986 (Patrick $\left.\operatorname{Bot}^{\ominus}\right)$
} 
verified. Then, simulations with different dock tunes and backstay loads highlight the importance of rig adjustments on the aerodynamic forces and the dynamic behaviour of a sail plan. The energy dissipated by the hysteresis is higher for looser shrouds and a tighter backstay.

Keywords: Fluid Structure Interaction, Dynamic behaviour, Yacht Sails, Pitching, Hysteresis, Parametric study 


\title{
NOMENCLATURE
}

\author{
A deg ${ }^{1} \quad$ pitching oscillation amplitude \\ $C \quad m \quad$ sail plan chord at $\mathrm{z}_{C E}$ (from head-sail leading edge to \\ mainsail trailing edge) \\ $C_{x} \quad$ driving force coefficient \\ $C_{y} \quad$ heeling force coefficient \\ $f_{r} \quad$ flow reduced frequency \\ $F_{x} \quad N \quad$ driving force \\ $F_{y} \quad N \quad$ side force \\ $M_{x} \quad$ N.m heeling moment \\ $M_{y} \quad$ N.m pitching moment \\ $P_{T O T} \quad W \quad$ total power of aerodynamic forces \\ $P_{L O O P} \quad W \quad$ dissipated power: power contained in the hysteresis loop \\ $P_{V_{B S}} \quad W \quad$ useful power: power driving the boat forward \\ $S \quad m^{2} \quad$ total sail area \\ $(O, X, Y, Z) \quad m \quad$ Inertial frame defined for an upright boat (origin $O$ at \\ the mast step, $X$ the yacht direction pointing forward, $Y$ \\ athwartships (upright) pointing portside (left), $Z$ vertical \\ pointing upwards) \\ $(O, x, y, z) \quad m \quad$ Boat frame defined for a pitched and heeled boat ( $x$ yacht \\ direction pointing forward, $y$ athwartships (heeled) pointing \\ portside (left), $z$ along mast pointing upwards) \\ $T \quad S \quad$ pitching oscillation period \\ $V_{A W} \quad m \cdot s^{-1}$ apparent wind speed \\ $V_{B S} \quad m \cdot s^{-1}$ boat speed
}




\begin{tabular}{|c|c|c|}
\hline$V_{T W}$ & $m . s^{-1}$ & true wind speed \\
\hline$V_{r}$ & & flow reduced velocity \\
\hline$Z_{C E}$ & $m$ & instantaneous altitude of the centre of aerodynamic forces \\
\hline$z_{C E}$ & $m$ & $\begin{array}{l}\text { in the inertial frame } \\
\text { instantaneous } z \text { coordinate of the centre of aerodynamic } \\
\text { forces in the boat frame (pitched and heeled) }\end{array}$ \\
\hline$\beta_{A W}$ & $\operatorname{deg}^{1}$ & apparent wind angle \\
\hline$\beta_{e f f}$ & $\operatorname{deg}^{1}$ & effective wind angle \\
\hline$\beta_{T W}$ & $\operatorname{deg}^{1}$ & true wind angle \\
\hline$\phi$ & $\operatorname{deg}^{1}$ & heel angle \\
\hline$\theta$ & $\operatorname{deg}^{1}$ & trim angle \\
\hline$\alpha$ & $\operatorname{deg}^{1}$ & heading angle \\
\hline$\rho$ & $k g \cdot m^{-3}$ & fluid density \\
\hline$\tau$ & $s$ & phase shift \\
\hline$\vec{F}$ & $N$ & \\
\hline$\vec{M}$ & $N . m$ & Aerodynamic force matrix: resultant and moment written in \\
\hline$\vec{\Omega}$ & $\operatorname{rad} . s^{-1}$ & $O$ \\
\hline$\vec{V}$ & $m \cdot s^{-1}$ & Boat kinematic matrix: rotation and velocity written in $O$ \\
\hline
\end{tabular}

\footnotetext{
${ }^{1}$ in degrees when a value is mentioned in the text, in radians in all formulae
} 


\section{Introduction}

When analysing the behaviour of yacht sails, an important difficulty comes from the Fluid Structure Interaction (FSI) of the air flow and the sails and rig (Fossati [2010], Garrett [1996], Marchaj [1996]). Yacht sails are soft structures whose shapes change according to the aerodynamic loading. The resulting modified shape affects the air flow and thus, the aerodynamic loading applied to the structure. This Fluid Structure Interaction is strong and non-linear, because sails are soft and light membranes which experience large displacements and accelerations, even for small stresses. As a consequence, the actual sail's shape while sailing — the so-called flying shape — is different from the design shape defined by the sail maker and is generally not known. Recently, several authors have focused on the Fluid Structure Interaction problem to address the issue of the impact of the structural deformation on the flow and hence the aerodynamic forces generated (Chapin and Heppel [2010], Renzsh and Graf [2010]).

Another challenging task in modelling racing yachts is to consider the yacht behaviour in a realistic environment (Charvet et al. [1996], Fossati [2010], Garrett [1996], Marchaj [1996]). Traditional Velocity Prediction Programs (VPPs) used by yacht designers consider a static equilibrium between hydrodynamic and aerodynamic forces. Hence, the force models classically used are estimated in a steady state. However, in realistic sailing conditions, the flow around the sails is most often largely unsteady because of wind variations, actions of the crew and more importantly because of yacht motion due to waves. To account for this dynamic behaviour, several Dynamic Velocity Prediction Programs (DVPPs) have been developed, (e.g. Keuning et al. [2005], Masuyama and Fukasawa [1997], Masuyama et al. [1993], Richardt et al. [2005]) which need models of dynamic aerodynamic 
and hydrodynamic forces. While the dynamic effects on hydrodynamic forces have been largely studied, the unsteady aerodynamic behaviour of the sails has received much less attention. Schoop and Bessert [2001] first developed an unsteady aeroelastic model in potential flow dedicated to flexible membranes but neglected the inertia. In a quasi-static approach, a first step is to add the velocity induced by the yacht's motion to the steady apparent wind to build an instantaneous apparent wind (see Keuning et al. [2005], Richardt et al. [2005]) and to consider the aerodynamic forces corresponding to this instantaneous apparent wind using force models obtained in the steady state. In a recent study, Gerhardt et al. [2011] developed an analytical model to predict the unsteady aerodynamics of interacting yacht sails in 2D potential flow and performed 2D wind tunnel oscillation tests with a motion range typical of a 90 -foot $(26 \mathrm{~m})$ racing yacht (International America's Cup Class 33). Recently, Fossati and Muggiasca [2009, 2010, 2011] studied the aerodynamics of model-scale rigid sails in a wind tunnel, and showed that a pitching motion has a strong and non-trivial effect on aerodynamic forces. They showed that the relationship between instantaneous forces and apparent wind deviates — phase shifts, hysteresis — from the equivalent relationship obtained in a steady state, which one could have thought to apply in a quasi-static approach. They also investigated soft sails in the same conditions to highlight the effects of the structural deformation (Fossati and Muggiasca [2012]).

In a previous work (Augier et al. [2013]), the aero-elastic behaviour of the sail plan subjected to a simple harmonic pitching was numerically investigated. This study has shown hysteresis phenomena between the aerodynamic forces and instantaneous apparent wind angle. A comparison between a rigid structure and a realistic soft structure showed that the hysteresis still exists for a rigid structure 
but it is lower than when the structure deformation is taken into account. However, in this first work (Augier et al. [2013]), the question whether this hysteresis could be represented by a simple phase shift between both oscillating signals was not clearly elucidated. Moreover, the energy exchange associated to the hysteresis phenomenon was not determined. Hence, the first aim of the present work is to investigate further this hysteresis phenomenon, to quantify the phase shift between aerodynamic forces and apparent wind angle, and to determine and analyse the associated energy.

Most of studies about the unsteady effect due to yacht pitching have considered a 2D simplified problem and thus approximated the pitching motion by a translational oscillation aligned with the yacht centreline (e.g. Fitt and Lattimer [2000], Gerhardt et al. [2011]). Then, the usual procedure is to decompose this surge motion in oscillations perpendicular to and along the direction of the incident flow, which results in oscillations of apparent wind angle and speed respectively (Fig.8). The second aim of this work is to investigate the effects of such simplifications in the yacht motion considered by comparing the results obtained with the sail plan subjected to different types of motion.

The third aim of this work is to address the effect of various rig and sail trims and adjustments commonly used by sailors on the unsteady aero-elastic behaviour of the sail plan subjected to pitching. This is investigated by comparisons of results obtained for realistic docktunes and backstay tensions used while racing a 28-foot ( $8 m$, J80 class) cruiser-racer.

An unsteady FSI model has been developed and validated with experiments in real sailing conditions (Augier et al. [2010, 2011, 2012]). Calculations are made on a J80 class yacht numerical model with her standard rigging and sails designed 
by the sail maker DeltaVoiles. The FSI model is briefly presented in section 2 . The methodology of the dynamic investigation is given in section 3 . In the continuity of a previous work (Augier et al. [2013]), section 4 gives further precisions on the dynamic behaviour with a particular attention to the energy exchange related to the hysteresis phenomenon. The analysis of pitching motion decomposition in simple translations is given in section 5 and the effects of various dock tunes and backstay loads are presented in sections 6.1 and 6.2. In the last section, some conclusions of this study are given, with ideas for future work.

\section{Numerical model}

To numerically investigate aero-elastic problems commonly found with sails, the company K-Epsilon and the Naval Academy Research Institute have developed the unsteady fluid-structure model ARAVANTI made by coupling the inviscid flow solver AVANTI with the structural solver ARA. The ARAVANTI code is able to model a complete sail boat rig in order to predict forces, tensile and shape of sails according to the loading in dynamic conditions. For more details, the reader is referred to Roux et al. [2002] for the fluid solver AVANTI and to Hauville et al. [2008] and Roux et al. [2008] for the structural solver ARA and the FSI coupling method.

ARAVANTI model has been validated. Numerical and experimental comparisons with the model ARAVANTI are based on measurements at full scale on an instrumented 28-foot yacht (J80 class, 8m). The time-resolved sails' flying shape, loads in the rig, yacht's motion and apparent wind have been measured in both sailing conditions of flat sea and moderate head waves and compared to the simulation. The code has shown its ability to simulate the rig's response to yacht 
motion forcing, and to correctly estimate the loads. Thereby, ARAVANTI is a reliable tool to study the dynamic behaviour of a sail plan subject to pitching motion. For a detailed description of the experimental system and the numerical and experimental comparison, see Augier et al. [2010, 2011, 2012].

\section{Simulation procedure}

The yacht motion in waves induces unsteady effects in the sails' aerodynamics. In this paper we will study separately one degree of freedom, by applying simple harmonic pitching. The reference frame and the coordinate system attached to the yacht are illustrated in Figure 1.

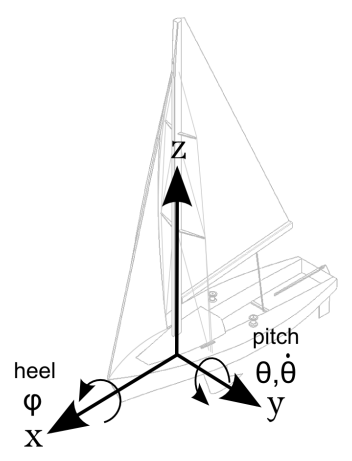

Figure 1: Coordinate, angle and motion references for the yacht. $\mathrm{Z}$ axis is attached to the earth vertical.

\subsection{Reference steady case}

First, the reference steady case is computed with the following parameters: true wind speed at $10 \mathrm{~m}$ height $V_{T W}=6.7 \mathrm{~m}_{\mathrm{s}} \mathrm{s}^{-1}$ (a logarithmic vertical wind profile is imposed with a roughness length of $0.2 \mathrm{~mm}$ (Flay [1996])), true wind angle $\beta_{T W}=40^{\circ}$, boat speed $V_{B S}=2.6 \mathrm{~m} . \mathrm{s}^{-1}$, heel angle $\phi=20^{\circ}$ and trim angle $\theta=0^{\circ}$. 


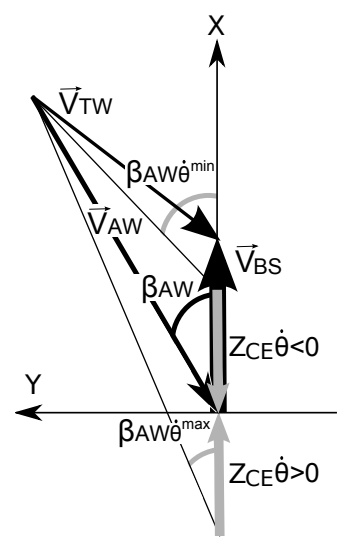

Figure 2: Dynamic effect of pitching on the wind triangle (top view). $\vec{V}$ is the wind velocity, $\overrightarrow{V_{B S}}$ is the boat speed, $Z_{C E}$ is the altitude of the aerodynamic centre of effort, $\dot{\theta}$ is the pitching velocity, $\beta$ is the apparent wind angle, subscripts $T W$ and $A W$ stand for True and Apparent Wind

This first computation yields the converged steady flow, the rig and sails' flying shape, and enables the steady state aerodynamic forces and centre of effort to be determined. The centre of effort is defined as the intersection of the boat symmetry plane with the aerodynamic forces matrix central axis, which is the line of points where the moment of aerodynamic forces is minimal (note that there is no point where this moment is exactly zero in general because the sails' shape is not developable). This converged steady state is used as the initial condition for the computations with pitching forcing. The height of the centre of aerodynamic forces $Z_{C E_{\text {Steady }}}=4.97 \mathrm{~m}$ is used to define the flow characteristic quantities: apparent wind speed $V_{A W}=8.39 \mathrm{~m} . \mathrm{s}^{-1}$, apparent wind angle $\beta_{A W}=28.64^{\circ}$ and sail plan chord $C=4.25 \mathrm{~m}$ defined as the distance from the head-sail leading edge to the main sail trailing edge at $Z_{C E_{\text {Steady }}}$.

Corrections of the apparent wind angle $\beta_{A W}$ due to constant heel $\phi$ (first introduced by Marchaj [1996]) and trim $\theta$ are considered through the use of the 

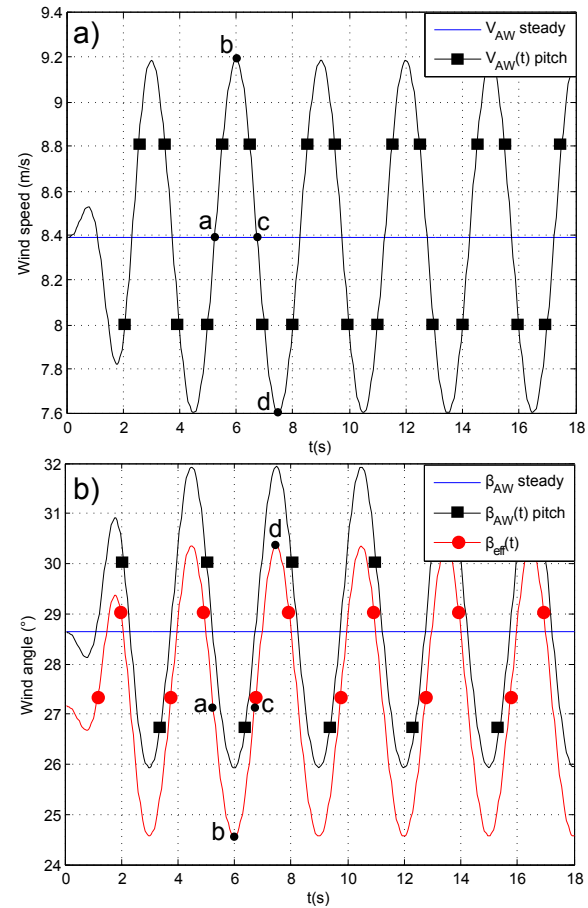

Figure 3: Time dependent apparent wind speed $V_{A W}$ (a); apparent wind angle $\beta_{A W}$ and effective wind angle $\beta_{\text {eff }}$ (b), resulting from pitching oscillation at $Z_{C E}$ with period $T=3 \mathrm{~s}$ and amplitude $A=5^{\circ}$. We define four reference points to be identified in further figures: bow up for point a $(\dot{\theta}=0, \theta=-A$ ), horizontal going down (no trim) for point $\mathrm{b}(\dot{\theta}>0, \theta=0)$, bow down for point $\mathrm{c}$ $(\dot{\theta}=0, \theta=A)$, horizontal going up (no trim) for point $\mathrm{d}(\dot{\theta}<0, \theta=0)$

effective apparent wind angle $\beta_{\text {eff }}$ (see Jackson [2001] for heel effect, and Fossati and Muggiasca [2011] for pitch effect):

$$
\beta_{e f f}=\tan ^{-1}\left(\frac{\tan \beta_{A W}}{\cos \theta} \cos \phi\right)
$$

$\beta_{\text {eff }}=27.16^{\circ}$ in the steady state. 


\subsection{Harmonic pitching}

The unsteady computations consist of a $18 s$ run, with forced harmonic pitching being imposed on the rig, characterised by the oscillation amplitude A and period $\mathrm{T}$ (equation 2), other parameters being constant and equal to those of the reference state.

$$
\theta=A \cos \left(\frac{2 \pi}{T} t\right)
$$

To avoid discontinuities in the accelerations, the beginning of motion is gradually imposed by applying a ramp which increases smoothly from 0 to 1 during the first $3 s$ of imposed motion (see first period in Figure 3). The investigation has been made with variables in the range $A=3$ to $6^{\circ}$, and $T=1.5$ to $6 s$, corresponding to the typical environmental conditions encountered, as shown in the experiment of Augier et al. [2012]. The unsteady nature of the flow is characterised by a dimensionless parameter defined by the ratio of the motion period $\mathrm{T}$ to the fluid advection time along the total sail plan chord C. Similarly to the closely related literature (e.g. Fossati and Muggiasca [2012], Gerhardt et al. [2011]), this parameter is called the flow reduced velocity $V_{r}$ (or the inverse: the reduced frequency $f_{r}$ ) defined by:

$$
V_{r}=\frac{V_{A W} T}{C} ; f_{r}=\frac{C}{V_{A W} T}
$$

The reduced frequency — which reads as a Strouhal number - was shown to be the relevant parameter to characterise the unsteadiness of lifting bodies aerodynamics (e.g. Abbott I. H. [1949], Gerhardt et al. [2011], Glauert [1926]). The 
case $V_{r} \gg 1\left(f_{r} \ll 1\right)$ corresponds to quasi-steady aerodynamic conditions. The pitching period values investigated correspond to a reduced velocity $V_{r}$ from 2.96 to 11.84 (reduced frequency $f_{r}$ from 0.08 to 0.34 ), which positions this numerical study in a similar dynamic range to the experiments of Fossati and Muggiasca [2011] where $V_{r}$ was from 2.3 to 56 (reduced frequency $f_{r}$ from 0.02 to 0.43 ) corresponding to typical conditions encountered by a 48 -foot yacht $(14.6 \mathrm{~m})$. The computed cases are summarised in Table 1.

When the yacht is subjected to pitching motion, the apparent wind is periodically modified as the rotation adds a new component of apparent wind which varies with height. Following the analysis of Fossati and Muggiasca [2011], the apparent wind and pitch-induced velocity are considered at the centre of aerodynamic force altitude $Z_{C E}$. This centre of effort is actually moving due to pitch oscillation, and the time-dependent centre of effort height is considered. This yields time-dependent apparent wind speed and angle, given by:

$$
\begin{aligned}
V_{A W}(t)= & \left(\left(V_{T W}\left(Z_{C E}\right) \sin \beta_{T W}\right)^{2}\right. \\
& \left.+\left(V_{T W}\left(Z_{C E}\right) \cos \beta_{T W}+V_{B S}+z_{C E} \dot{\theta} \cos \theta \cos \phi\right)^{2}\right)^{\frac{1}{2}} \\
\beta_{A W}(t)= & \sin ^{-1}\left(\frac{V_{T W} \sin \beta_{T W}}{V_{A W}(t)}\right)
\end{aligned}
$$

And hence the time-dependent effective wind angle reads:

$$
\beta_{e f f}(t)=\tan ^{-1}\left(\frac{\tan \beta_{A W}}{\cos \theta} \cos \phi\right)
$$

Figure 2 illustrates the dynamic vector composition for pitching velocities $\dot{\theta}=$ $\dot{\theta}_{\max }$ (point b in Fig. 3), 0 (point a and c in Fig. 3) and $\dot{\theta}_{\text {min }}$ (point d in Fig. 3), and 
Figure 3 shows the resulting dynamic apparent wind velocity and angle computed with equations 4 and 5. As shown in Figure 3, the variations of the apparent wind angle are in phase opposition with the variations of apparent wind speed.

\subsection{Heeling and driving force coefficients}

Aerodynamic forces are computed by ARAVANTI as the resultant of pressures on the sails and the aerodynamic forces matrix (resultant and moment) is written at the origin 0 in the inertial frame illustrated Fig. 1.

A transition matrix $\left[R_{T}\right]$ can be used in order to get forces in the boat frame using the following equation $\vec{F}_{\text {Boat frame }}=\left[R_{T}\right]^{-1} \vec{F}_{\text {Inertial frame }}$ The transition matrix $\left[R_{T}\right]$ is defined by $\left[R_{T}\right]=\left[R_{\alpha}\right]\left[R_{\theta}\right]\left[R_{\phi}\right]$ with:

$$
\left[R_{\phi}\right]=\left[\begin{array}{ccc}
1 & 0 & 0 \\
0 & \cos \phi & -\sin \phi \\
0 & \sin \phi & \cos \phi
\end{array}\right],\left[R_{\theta}\right]=\left[\begin{array}{ccc}
\cos \theta & 0 & \sin \theta \\
0 & 1 & 0 \\
-\sin \theta & 0 & \cos \theta
\end{array}\right],\left[R_{\alpha}\right]=\left[\begin{array}{ccc}
\cos \alpha & -\sin \alpha & 0 \\
\sin \alpha & \cos \alpha & 0 \\
0 & 0 & 1
\end{array}\right]
$$

Driving and heeling force coefficients in the boat frame are obtained by the normalisation with the product of the instantaneous apparent dynamic pressure and the total sail area $S$ :

$$
\begin{aligned}
C_{x}(t) & =\frac{F_{x}}{0.5 \rho V_{A W}^{2}(t) S} \\
C_{y}(t) & =\frac{F_{y}}{0.5 \rho V_{A W}^{2}(t) S}
\end{aligned}
$$

In the steady state calculation, driving force coefficient $C_{x}=0.423$ and heeling force coefficient $C_{y}=-1.080$ are obtained. 


\section{Dynamic behaviour}

Previous studies (Augier et al. [2013], Fossati and Muggiasca [2011]) have shown that the dynamic behaviour of a yacht sail plan subjected to pitching clearly deviates from the quasi-static approach. Particularly, the aerodynamic forces presented as a function of the instantaneous apparent wind angle show hysteresis loops as illustrated in figure 4. Different questions have been raised by this result. (i) Can the loops in the Lissajous plots be represented by a simple phase shift between the signals? This hysteresis phenomenon suggests that the unsteady behaviour leads to aerodynamic equivalent damping and stiffening effects. The area included in the hysteresis loop was shown to increase with the motion reduced frequency and amplitude, but the exchanged energy was not investigated. (ii) Can the energy associated to the hysteresis be determined and analysed for different pitching frequencies and amplitudes?

\subsection{Phase shift $\tau$}

The values of the phase shift $\tau$ between aerodynamic forces and instantaneous wind angle have been determined for each pitching period and amplitude by crosscorrelation (Table 1). The phase delay $2 \pi \tau / T$ in radians increases (almost linearly in the investigated range) with the flow reduced velocity, meaning with the motion period, but is not affected by the oscillation amplitude. When force coefficients $C_{x, y}(t)$ are plotted versus the time shifted wind angle $\beta_{e f f}(t+\tau)$, the loop area is significantly decreased but does not vanish (see Fig. 5). Moreover, as shown on Fig. 4 and 6, the loops are not purely elliptical because of non-linear effects. This

shows that the hysteresis phenomenon cannot be reduced to a simple phase shift between the signals. 


\begin{tabular}{|c|c|c|c|c|c|c|c|c|c|c|}
\hline $\begin{array}{l}T \\
\text { (s) }\end{array}$ & $\begin{array}{c}A \\
(\mathrm{deg})\end{array}$ & $V_{r}$ & $f_{r}$ & $\begin{array}{c}\tau \\
(\mathrm{s})\end{array}$ & $\begin{array}{r}2 \pi \tau / T \\
(\mathrm{rad})\end{array}$ & $\begin{array}{c}\overline{P_{T O T}} \\
(\mathrm{~W})\end{array}$ & $\begin{array}{c}\overline{P_{L O O P}} \\
(\mathrm{~W})\end{array}$ & $\begin{array}{l}\overline{P_{V_{B S}}} \\
(\mathrm{~W})\end{array}$ & $\begin{array}{l}\overline{F_{X}} \\
(\mathrm{~N})\end{array}$ & $\begin{array}{c}\overline{M_{X}} \\
\text { (N.m) }\end{array}$ \\
\hline 1.5 & 5 & 2.96 & 0.34 & 0.16 & 0.670 & 1454 & -106.43 & 1561 & 608.8 & 8290 \\
\hline 2 & 5 & 3.95 & 0.25 & 0.29 & 0.921 & 1518 & -55.57 & 1574 & 613.3 & 8274 \\
\hline 2.5 & 5 & 4.94 & 0.20 & 0.50 & 1.257 & 1540 & -35.60 & 1576 & 614.1 & 8244 \\
\hline 3 & 5 & 5.92 & 0.17 & 0.76 & 1.592 & 1558 & -24.89 & 1583 & 616.3 & 8260 \\
\hline 5 & 5 & 9.87 & 0.10 & 2.70 & 3.393 & 1580 & -9.98 & 1590 & 618.5 & 8266 \\
\hline 6 & 5 & 11.84 & 0.08 & 4.12 & 4.314 & 1584 & -7.37 & 1592 & 619.1 & 8270 \\
\hline $\begin{array}{l}T \\
\text { (s) }\end{array}$ & $\begin{array}{c}A \\
(\mathrm{deg})\end{array}$ & $V_{r}$ & $f_{r}$ & $\begin{array}{l}\tau \\
(\mathrm{s})\end{array}$ & $\begin{array}{r}2 \pi \tau / T \\
(\mathrm{rad})\end{array}$ & $\begin{array}{r}\overline{P_{T O T}} \\
(\mathrm{~W})\end{array}$ & $\begin{array}{c}\overline{P_{L O O P}} \\
(\mathrm{~W})\end{array}$ & $\begin{array}{l}\overline{P_{V_{B S}}} \\
(\mathrm{~W})\end{array}$ & $\begin{array}{l}\overline{F_{X}} \\
(\mathrm{~N})\end{array}$ & $\begin{array}{c}\overline{M_{X}} \\
\text { (N.m) }\end{array}$ \\
\hline 5 & 3 & 9.87 & 0.10 & 2.70 & 3.393 & 1588 & -3.63 & 1591 & 619.1 & 8262 \\
\hline 5 & 5 & 9.87 & 0.10 & 2.70 & 3.393 & 1580 & -9.98 & 1590 & 618.5 & 8266 \\
\hline 5 & 6 & 9.87 & 0.10 & 2.70 & 3.393 & 1574 & -14.44 & 1589 & 618.0 & 8268 \\
\hline
\end{tabular}

Table 1: Reduced velocity $V_{r}$, reduced frequency $f_{r}$, phase shift $\tau$ determined by cross-correlation between $C_{x}$ and $\beta_{e f f}$, phase delay, time-averaged total power $\overline{P_{T O T}}$, time-averaged dissipated power $\overline{P_{L O O P}}$, time-averaged useful power $\overline{P_{V_{B S}}}$, time-averaged driving force $\overline{F_{X}}$, and time-averaged heeling moment $\overline{M_{X}}$ for different pitching amplitudes $A$ and periods $T$ 

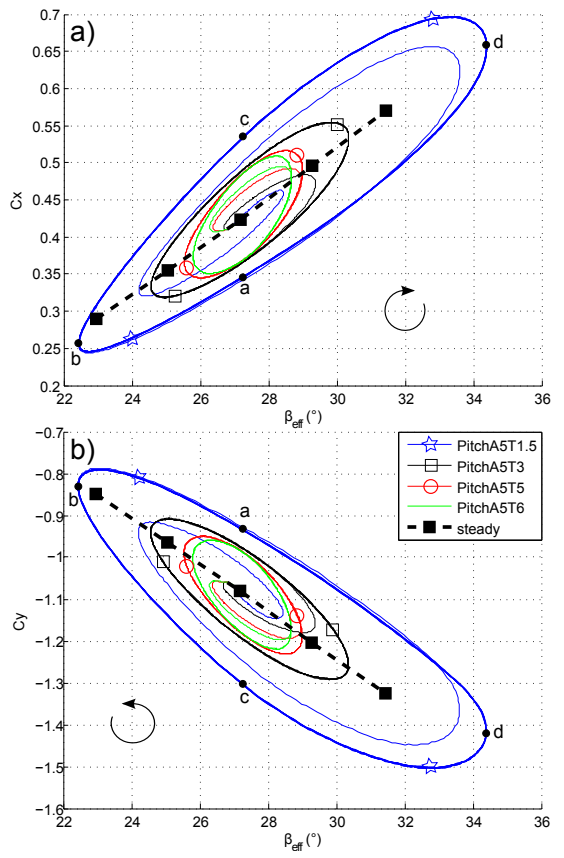

Figure 4: Driving a) and heeling b) force coefficients versus effective wind angle $\beta_{\text {eff }}(\mathrm{t})$.

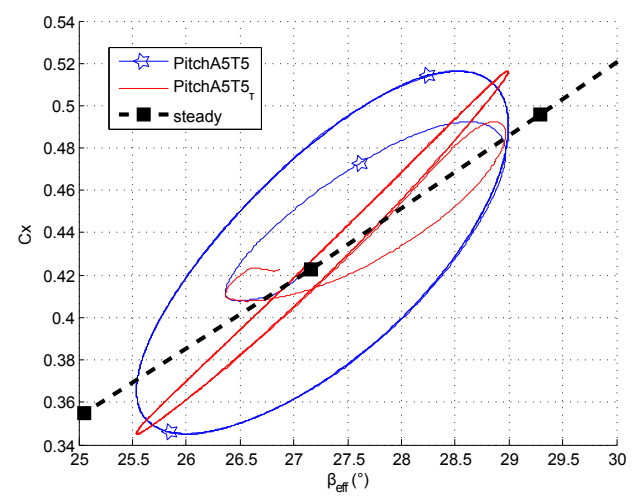

Figure 5: Driving force coefficient vs. instantaneous apparent wind angle $\beta_{\text {eff }}(t)$ (blue line with markers), and vs. the time shifted instantaneous apparent wind angle $\beta_{e f f}(t+\tau)$ (red line without marker), for a pitching period $T=5 \mathrm{~s}$ and amplitude $A=5^{\circ}$ 


\subsection{Exchanged Energy}

The hysteresis phenomenon observed in the aerodynamics of the pitching sail plan corresponds to an exchange of energy between the yacht motion and the aeroelastic system. The aim of this section is to determine and analyse this energy for different values of the motion parameters. Indeed, the energy per unit time is considered, i.e. the exchanged power, which is more relevant to compare different motion frequencies. The area contained in the hysteresis loop of Fig. 4 does not formally correspond to an energy nor a power as $\beta_{\text {eff }}$ is the effective apparent wind angle and its relationship to a displacement or velocity is not straightforward.

The dimensional energy in Joules — or dimensional power in Watts - is considered instead of dimensionless quantities to avoid biased effects introduced by normalizing with the varying dynamic pressure.

The instantaneous mechanical power is defined by its general expression combining the kinematic and dynamic matrices:

$$
P_{\text {TOT }}(t)=\vec{F} \cdot \vec{V}+\vec{M} \cdot \vec{\Omega}
$$

where "." denotes the scalar product between vectors. For the motion considered in this work (forward translation and pitching), this expression reduces to:

$$
P_{T O T}(t)=F_{X} V_{B S}+M_{Y} \dot{\theta}
$$

The first term on the right hand side $F_{X} V_{B S}=P_{V_{B S}}$ is the useful power driving the yacht forward. The second term $M_{Y} \dot{\theta}=P_{L O O P}$ is the power exchanged by the system due to the hysteresis phenomenon.

Figure 6 shows the aerodynamic force pitching moment $M_{Y}$ as a function of the trim angle $\theta$ for different values of the oscillation period $T$ from 1.5 up to 


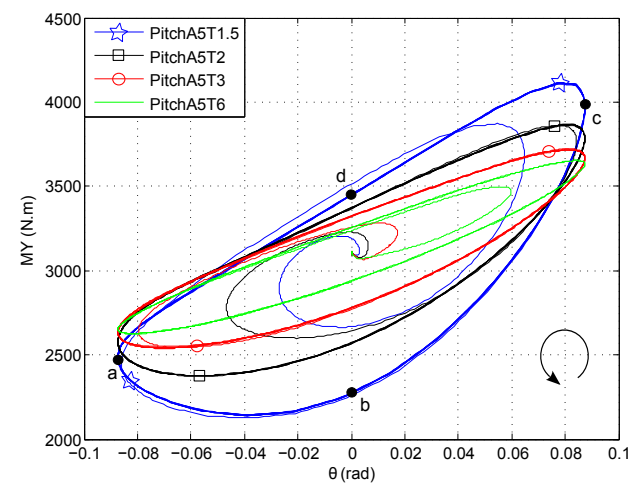

Figure 6: Pitching moment $M_{Y}$ vs. pitch angle $\theta$ for pitching periods $T=1.5,2,3,6 s$. The loop area represents the energy dissipated during the corresponding period ( $T$ times $\overline{P_{L O O P}}$ ).

$6 s$ with a pitching amplitude $A=5^{\circ}$. The area contained in these loops is the energy exchanged during one oscillation period between the system and the imposed pitching motion due to the hysteresis phenomenon. As shown by the rotation direction in the loops (Fig. 6) and the computed results (Table 1), this quantity is negative which means that some energy is dissipated by the hysteresis phenomenon. In the following, the mean power averaged over one oscillation period is considered:

$$
\begin{gathered}
\overline{P_{T O T}}=\frac{1}{T} \int_{T} P_{T O T} d t \\
\overline{P_{V_{B S}}}=\frac{1}{T} \int_{T} P_{V_{B S}} d t=\overline{F_{X}} V_{B S} \\
\overline{P_{L O O P}}=\frac{1}{T} \int_{T} P_{L O O P} d t=\frac{1}{T} \int_{T} M_{Y} \dot{\theta} d t
\end{gathered}
$$

$\overline{P_{V_{B S}}}$ is the useful mean power driving the boat forward and extracted from the 
air flow by the sail plan. $\overline{P_{V_{B S}}}$ is proportional to the mean driving force $\overline{F_{X}}$ as the boat speed is constant.

$\overline{P_{L O O P}}$ is the mean power dissipated by the hysteresis phenomenon from the imposed pitching motion and corresponds to the loops area on Fig. 6 divided by the pitching period $T$. At first order, this quantity is dominated by $\int_{T} F_{X} Z_{C E} \dot{\theta} d t$.

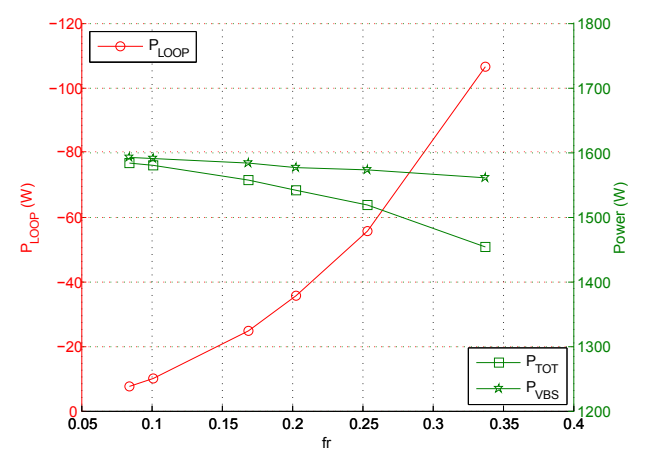

Figure 7: Dissipated power $\overline{P_{L O O P}}$, useful power $\overline{P_{V_{B S}}}$ and total power $\overline{P_{T O T}}$ for different reduced frequency $f_{r}$.

Note that the pitching motion itself introduces an added power to the system compared to the steady case (no pitching).

As shown on Fig. 7, the dissipated average power absolute value $\left|\overline{P_{L O O P}}\right|$ strongly increases with the motion reduced frequency, from zero for the steady case (vanishing frequency) up to $106 \mathrm{~W}$ for $f_{r}=0.34$. The non-linearity of the phenomenon is highlighted by the observation that the loop shape becomes distorted for the highest values of the reduced frequency (Fig. 6). The mean useful power $\overline{P_{V_{B S}}}$ decreases slightly (about $2 \%$ in the investigated range) for an increasing frequency, suggesting a small reduction of aerodynamic efficiency for a faster pitching motion.

Moreover, the mean driving force $\overline{F_{X}}$ is different from the driving force in the 
steady case $F_{X}$ steady as aerodynamic forces in the dynamic regime do not follow the quasi-static assumption and some power is exchanged due to the hysteresis. The total mean power $\overline{P_{T O T}}$ decreases more (about $8 \%$ in the investigated range) as the dissipated energy is higher in absolute value. As shown in Table 1, the effect of the pitching amplitude yields similar trends than the reduced frequency, i.e. increasing $\left|\overline{P_{L O O P}}\right|$, decreasing $\overline{P_{V_{B S}}}, \overline{F_{X}}$ and $\overline{P_{T O T}}$ for a higher pitching amplitude. It shall be noticed that $\overline{P_{T O T}}$ and $\overline{P_{V_{B S}}}$ are one to two orders of magnitude higher than $\left|\overline{P_{L O O P}}\right|$, which means that the useful power $\overline{P_{V_{B S}}}$ is dominant.

The aerodynamic behaviour is now clearly characterised: an hysteresis phenomenon is evidenced and the associated energy is analysed. The next sections address the various influences of the yacht motions considered and of different rig trims.

\section{Pitching decomposition}

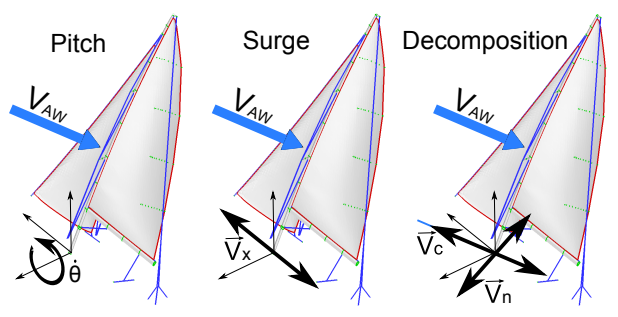

Figure 8: Different motions considered: pitching (rotation), surge (translation), surge decomposition into translations collinear to the apparent wind $V_{c}$ and normal to the apparent wind $V_{n}$.

The real pitching motion is modelled in this work by an angular oscillation around the $Y$ axis (Fig.8 Pitch), normal to the centreline with a rotation centre located at the mast step. Most of previous studies on the influence of pitching have 
considered a 2D simplified problem and thus approximated the pitching motion by a translational oscillation aligned with the yacht centreline (Fig.8 Surge). Then, the usual procedure (see e.g. Gerhardt et al. [2011]) is to decompose this motion in an oscillation parallel to the apparent wind, resulting in an oscillation of apparent wind speed, and an oscillation orthogonal to the apparent wind, resulting mainly in an oscillation of the apparent wind angle (Fig.8 decomposition). Here, we want to test these two hypotheses by comparing the results of the dynamic simulation with ARAVANTI obtained with different imposed motions, and investigate the effect on the specific dynamic features highlighted above. Motions are based on the reference pitching motion with amplitude $A=5^{\circ}$ and period $T=5 \mathrm{~s}$ (A5T5).

\subsection{Surge}

The first step is to compare the results for a real pitching motion (rotation) to the results for a translational surge motion with the amplitude of motion at the centre of effort height $Z_{C E}$ while pitching. As shown on Fig. 9 the oscillation of aerodynamic forces is decreased by 30 to $40 \%$ and phase shifted (around $T / 9$ ) when the pitching is reduced to a surge motion. This result gives the order of the error on the oscillation amplitude of aerodynamic forces introduced by considering a surge motion instead of the pitching motion.

Concerning the dynamic behaviour, it is interesting to notice that the case of surge does not show the same hysteresis phenomenon. Indeed, the aerodynamic behaviour in the case of surge is much closer to the quasi-steady theory than in the

pitching case, as clearly shown on Fig 10 . The loops of $C_{x, y}\left(\beta_{e f f}\right)$ collapse and are superposed to the steady case line. 

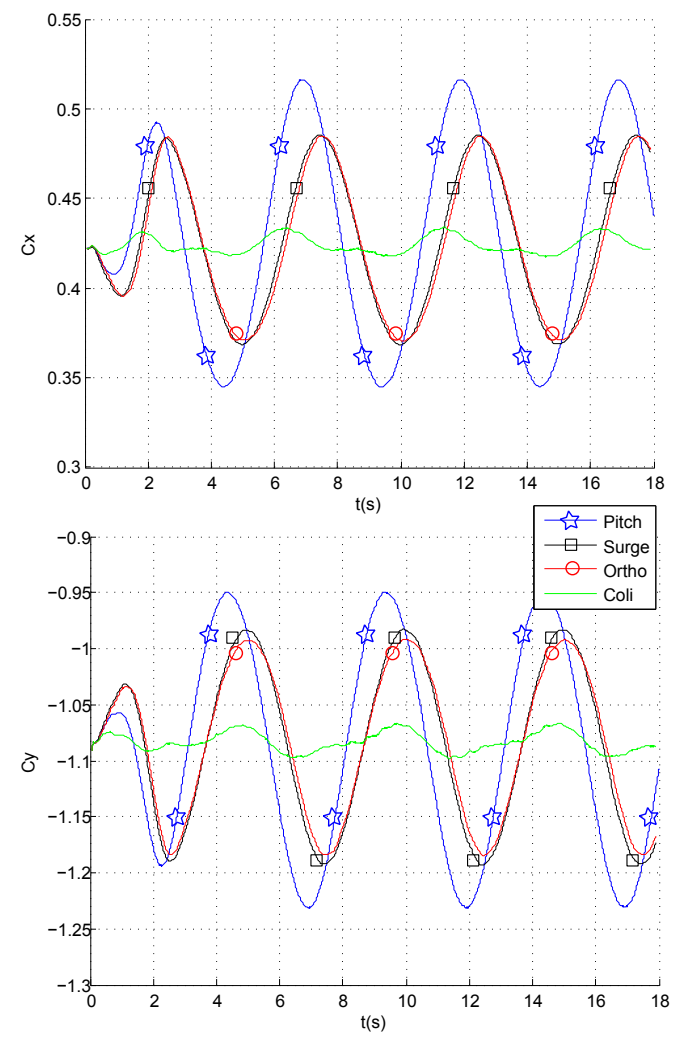

Figure 9: Time series of the driving and heeling force coefficients for FSI simulations of the various motions considered: pitching, surge, translations collinear and perpendicular to the apparent wind (see Fig.11), corresponding to a pitching amplitude $A=5^{\circ}$ and period $T=5 \mathrm{~s}$.

\subsection{Simple translations decomposition}

The second step is to analyse separately the effects of translational oscillations parallel $V_{c}$ (Fig. 11.a) and orthogonal $V_{n}$ (Fig 11.b) to the apparent wind direction. It is observed on Fig. 9 that the major contribution to the force oscillation is due to the orthogonal oscillation component, which is associated to the oscillation of apparent wind angle. When the variations due to both components of motion are added as shown on Fig. 12, the result is identical to what is obtained with the 

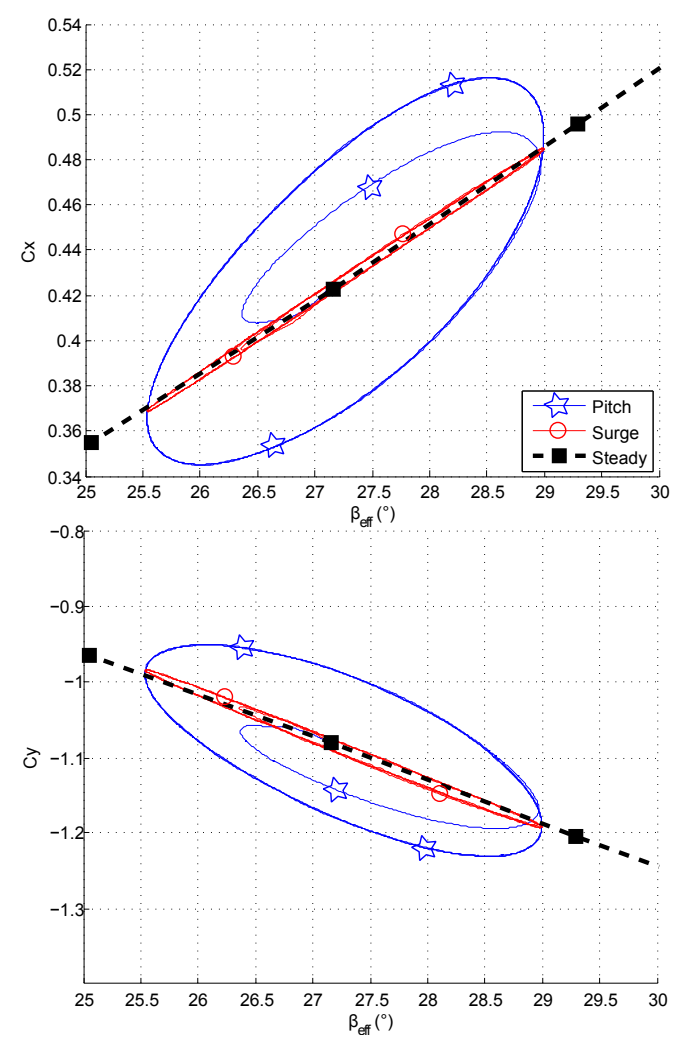

Figure 10: Driving and heeling force coefficients versus apparent wind angle for pitch and surge motions. The motion period and amplitude at the centre of effort are identical and correspond to a pitching amplitude $A=5^{\circ}$ and period $T=5 \mathrm{~s}$.

surge motion as both curves are superimposed, which justifies the linear superposition principle of this approach. The effect of parallel oscillation — variation of $V_{A W}(t)$ - is small, but with a more distorted evolution.

Note that the orthogonal oscillation is associated to an oscillation of $\beta_{A W}(t)$, and the effect of angle of attack in a narrow range is almost linear on the aerodynamic lift. Contrarily, the parallel oscillation is associated with an oscillation of $V_{A W}(t)$, and the effect of wind speed is quadratic on aerodynamic forces. 

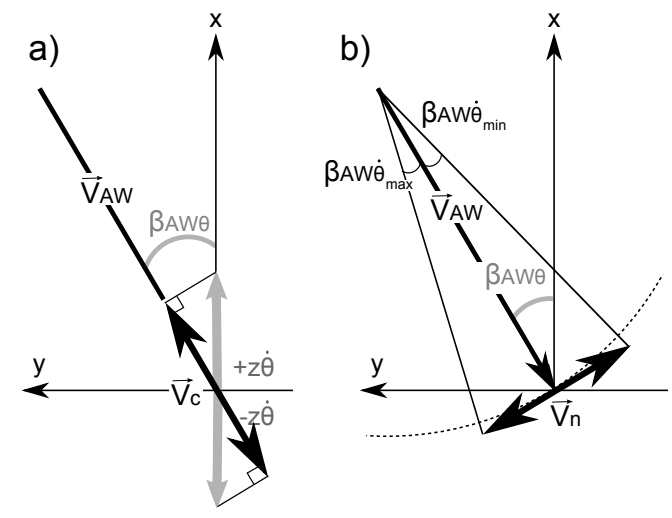

Figure 11: Wind triangle representation for the surge decomposition into 2 translations a) $V_{c}$ collinear to $V_{A W}$ and b) $V_{n}$ normal to $V_{A W}$.

\section{Influence of rig adjustments}

Before each race, sailors adjust the tension in the shrouds (dock tune) according to sailing conditions, and the backstay tension is often adjusted continuously while sailing upwind. In this section, the analysis of the effects of various dock tunes and backstay loads on the dynamic behaviour and the exchanged energy is presented.

\subsection{Influence of dock tune}

The influence of various dock tunes on the sail plan dynamic behaviour is investigated. The reference pitching motion $\left(A=5^{\circ}\right.$ and $\left.T=1.5 \mathrm{~s}\right)$ is simulated with three realistic dock tunes used while racing in different wind conditions. Dock tunes are defined as the number of screw turns applied to the shrouds' turnbuckles. Tune 2 is the reference dock tune used for the considered sailing conditions. The three dock tunes are described bellow:

- tune 1 : -3 turns on V1 shrouds used in light wind 

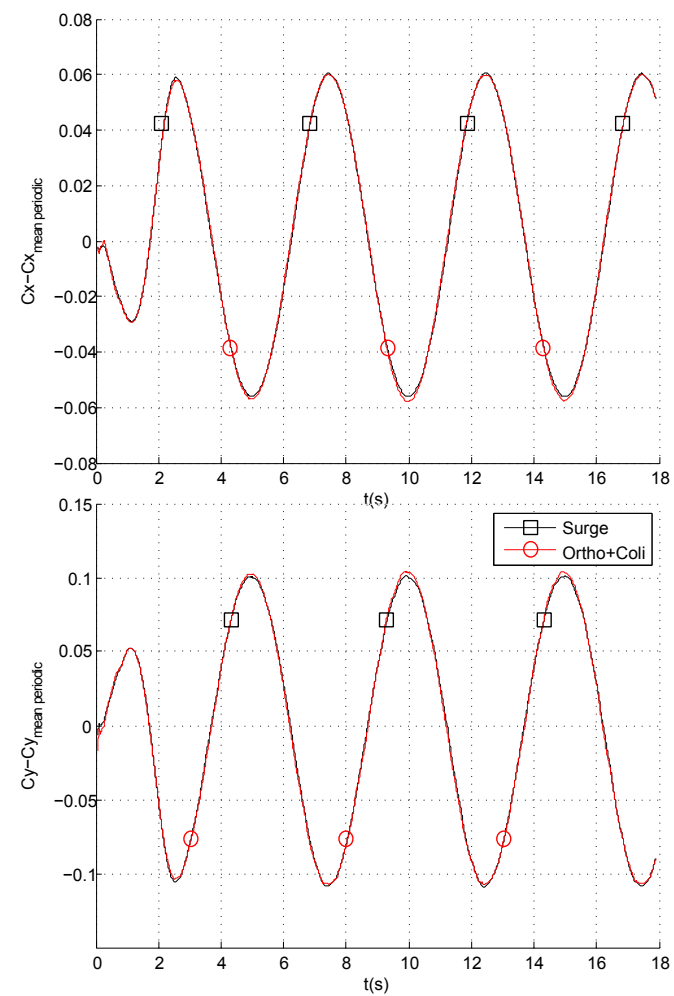

Figure 12: Comparison of oscillations of aerodynamic force coefficients obtained for a surge motion with the sum of oscillations obtained for both translation components parallel and orthogonal to the apparent wind.

- tune 2 : reference dock tune for $V_{T W}=6.7 \mathrm{~m}_{\mathrm{s}} \mathrm{s}^{-1}$ (13 knots)

- tune $3:+3$ turns on V1 shrouds used in medium wind

where V1 are the outer and highest lateral shrouds. The other shrouds are not modified.

This three dock tunes not only modify the rigidity of the full rigging but have a significant influence on the camber of the mast. Increasing the V1 tension makes a stiffer rig, a reduced forestay sag and a more bent mast, which results in flatter 
sails. The sails' shape and more precisely their camber and twist are modified by the dock tune. Before the pitching simulation, the main sail and jib are numerically trimmed in order to ensure that the chord at the centre of effort height has the same angle of attack for the different tunes to get a relevant comparison.

Figure 13 shows the energy loops of pitching moment $M_{Y}$ versus pitch angle for the three tested dock tunes. The loops look similar, however, the exchanged energy computed as described in section 4 shows variations. Table 2 presents the relative evolution of the mean total power $\overline{P_{T O T}}$, dissipated power $\overline{P_{L O O P}}$ and useful power $\overline{P_{V_{B S}}}$ which is equivalent to the average drive force $\overline{F_{X}}$. Compared to the reference dock tune 2 , the dissipated power is increased by $8.5 \%$ for the loosest rig and similar for the tightest rig. The reduction of dissipated energy with the increase of rig tension seems to be due to a stiffer rig. With more stresses, the rig is getting closer to a rigid structure and comparison between FSI and rigid simulations has shown that the hysteresis phenomenon is significantly lower in the rigid case (Augier et al. [2013]). Another factor may be that flatter sails dissipate less power.

The useful power is slightly higher (1.3\%) for the loosest rig and lower (2\%) for the tightest rig. This would suggest that the reference dock tune 2 is not optimal and a looser rig would be faster. However, it shall be recalled that this simulation is based on an inviscid flow which is known to find a higher drive force for sails with more camber than the real optimum because flow separation is not modelled. As a looser rig results in more cambered sails, this may be the reason why the mean useful power, or mean driving force, is predicted to be higher for tune 1 than for tune 2. Moreover, a performance analysis should also consider the side force, and the evolution of the mean heeling moment $\overline{M_{X}}$ is also given in 


\begin{tabular}{ccccc}
\hline dock tune & $\overline{\overline{P_{T O T}}}$ & $\overline{\overline{P_{L O O P}}}$ & $\overline{\overline{P_{V_{B S}}}}$ & $\overline{\overline{M_{X}}}$ \\
tune $_{1}$ & 1.008 & 1.085 & 1.013 & 1.017 \\
tune $_{2}$ & 1 & 1 & 1 & 1 \\
tune $_{3}$ & 0.979 & 0.999 & 0.980 & 0.977 \\
\hline
\end{tabular}

Table 2: Mean total power $\overline{P_{T O T}}$, mean dissipated power $\overline{P_{L O O P}}$, mean useful power $\overline{P_{V_{B S}}}$ and mean heeling moment $\overline{M_{X}}$ for different dock tunes, relative to reference case (tune ${ }_{2}$ ), for a pitching amplitude $A=5^{\circ}$ and period $T=1.5 \mathrm{~s}$.

Table 2 for information.

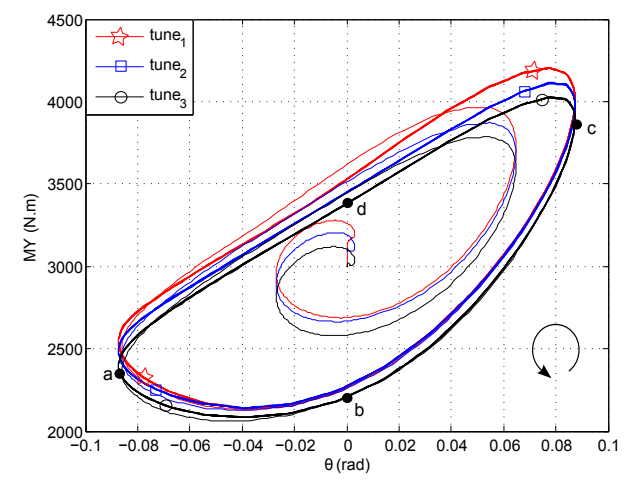

Figure 13: Pitching moment $M_{Y}$ vs. pitch angle $\theta$ for different dock tunes, for a pitching amplitude $A=5^{\circ}$ and period $T=1.5 \mathrm{~s}$. The loop area represents the energy dissipated during the corresponding period ( $T$ times $\overline{P_{L O O P}}$ ).

\subsection{Influence of the backstay load}

The influence of a variation of the backstay tension on the dynamic behaviour is investigated. The same pitching motion $\left(A=5^{\circ}\right.$ and $\left.T=1.5 \mathrm{~s}\right)$ is simulated with four values of backstay length corresponding to backstay loads of $1000 \mathrm{~N}$, $1500 N, 2000 N$ and $2500 N$ in the steady case. The case $2000 N$ is the reference 
backstay load used for the previous simulations. The sail trims are identical for the four backstay loads.

Preliminary steady simulations with the four loads have shown the ability of ARAVANTI model to simulate the effect of the backstay: the mainsail twist increases, the mainsail camber decreases and moves backward when the backstay load increases.

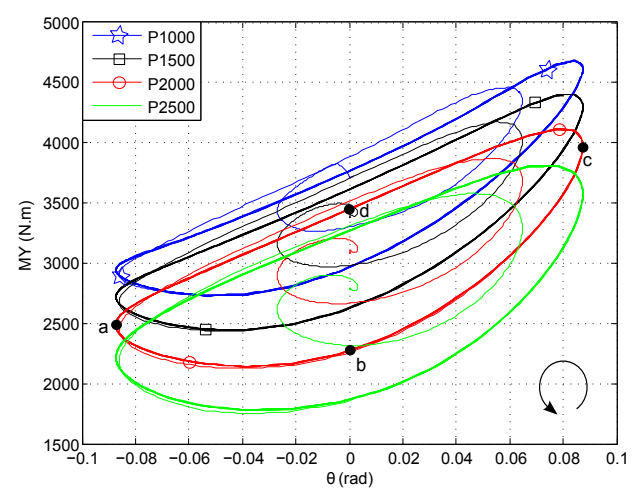

Figure 14: Pitching moment $M_{y}$ vs. pitch angle $\theta$ for different backstay loads, for a pitching amplitude $A=5^{\circ}$ and period $T=1.5 \mathrm{~s}$. The loop area represents the energy dissipated during the corresponding period ( $T$ times $\overline{P_{L O O P}}$ ).

Figure 14 shows the energy loops of pitching moment $M_{Y}$ versus pitch angle for different values of the backstay load. As expected, the mean driving force $\overline{F_{X}}$ (which is proportional to $\overline{P_{V_{B S}}}$ ) and the mean heeling moment $\overline{M_{X}}$ are greatly affected by the backstay load, which changes the main sail camber and twist (see Tab. 3).

Similarly to what is shown in Section 6.1, the lowest backstay load looks to be optimal in terms of mean driving force or useful power. Once again, the same restriction must be made due to the inviscid flow model which may bias the op- 


\begin{tabular}{ccccc}
\hline load & $\frac{\overline{P_{T O T}}}{\overline{P_{T O T} r e f}}$ & $\frac{\overline{P_{L O O P}}}{\overline{P_{L O O P} r e f}}$ & $\frac{\overline{P_{V_{B S}}}}{\overline{P_{B S}}}$ & $\frac{\overline{M_{X}}}{\overline{M_{X} r e f}}$ \\
$1000 \mathrm{~N}$ & 1.174 & 0.686 & 1.140 & 1.198 \\
$1500 \mathrm{~N}$ & 1.088 & 1.072 & 1.052 & 1.098 \\
$2000 \mathrm{~N}$ & 1 & 1 & 1 & 1 \\
$2500 \mathrm{~N}$ & 0.895 & 1.211 & 0.916 & 0.890 \\
\hline
\end{tabular}

Table 3: Mean total power $\overline{P_{T O T}}$, mean dissipated power $\overline{P_{L O O P}}$, mean useful power $\overline{P_{V_{B S}}}$ and mean heeling moment $\overline{M_{X}}$ for different backstay loads, relative to reference case $(2000 \mathrm{~N})$, for a pitching amplitude $A=5^{\circ}$ and period $T=1.5 \mathrm{~s}$.

timisation. Moreover, the mean heeling moment is $20 \%$ higher for the loosest backstay. This is consistent with the sailors knowledge who commonly tighten the backstay to reduce heel.

The backstay load also has a great influence on the energy dissipated in the hysteresis loop (see Tab. 3). The computed mean dissipated power strongly increases when the backstay load is increased $\left(\left|\overline{P_{L O O P}}\right|\right.$ almost doubles when the backstay is tighten from $1000 \mathrm{~N}$ up to $2500 \mathrm{~N}$ ). It is worth noticing that this trend is opposite to the one observed for a tighter dock tune being closer to a rigid structure as shown in Section 6.1. In the present case, more tension on the backstay results in flatter sails, but the main sail leech is also looser. This may result in more flapping of the main sail while pitching which can dissipate more power.

\section{Conclusions}

The unsteady fluid structure interaction of the sails and rig of a 28 -foot $(8 \mathrm{~m})$ yacht under harmonic pitching has been investigated in order to highlight the contributions of the rig adjustments and the consideration of a realistic pitching mo- 
tion in the dynamic behaviour of a sail plan. The ARAVANTI model is based on an implicit unsteady coupling between a vortex lattice fluid model and a finite element structure model, and has been previously validated with full scale experiments in upwind real conditions (Augier et al. [2012]). Previous studies (Augier et al. [2013], Fossati and Muggiasca [2012]) have shown that the aerodynamic coefficients plotted against the instantaneous apparent wind angle exhibit an hysteresis loop. The present results confirm that the dynamic behaviour of a sail plan subject to yacht motion deviates from the quasi-steady theory and an aerodynamic equivalent damping effect is highlighted. Oscillations of the aerodynamic forces exhibit an hysteresis phenomenon which increases with the motion reduced frequency and amplitude.

In this article, it is shown that the hysteresis loop area is not only due to a phase shift between the signals. After shifting by the phase delay $\tau$, the hysteresis loop of $\mathrm{C}_{x, y}=f\left(\beta_{e f f}(t+\tau)\right)$ does not collapse into a single line.

The power of aerodynamic forces is investigated and analysed in terms of useful power and power exchanged between the system and motion through the hysteresis phenomenon. It is shown that some energy is dissipated by the aeroelastic system from the energy input by the motion. This dissipated energy increases with the motion reduced frequency and amplitude. The useful energy associated to the driving force is lower for a faster and higher amplitude pitching motion. The motion considered in this work is a constant boat speed and forced pure harmonic pitching only, and all other degrees of freedom are kept constant. In reality, when the aerodynamic forces oscillate, the heel angle vary accordingly, and to a smaller extent the boat speed and leeway, so other terms must be considered in the expression of power. Further work is needed to investigate the effect of other 
types of motion on the exchanged energy. It would be interesting to try and find some favourable motion resulting in a higher useful power and mean driving force than the steady case. From sailors experience who sometimes force a rolling motion, called rocking, we expect that this may be obtained for a properly chosen roll motion of the rig. This interesting behaviour would resemble a flapping wing producing thrust.

Pure harmonic surge motion is compared to pitching motion in order to highlight the importance of a realistic 3D motion. Oscillations of the aerodynamic coefficients decrease by 30 to $40 \%$ in the case of an equivalent surge motion compared to the pitching motion case. Moreover, in the case of the surge motion, the hysteresis phenomenon is almost cancelled, so that the dynamic behaviour is similar to the quasi-steady theory. When the surge motion is decomposed into two components, perpendicular to and along the apparent wind direction, it is shown that the major contribution to force oscillations is due to the orthogonal oscillation component, which is associated to the oscillation of apparent wind angle.

Finally, a pitching motion of the structure with various shrouds' dock tunes and backstay tension loads is simulated in order to study the influence of the rigging stresses on the dynamic behaviour.

Tighter shrouds resulting in flatter sails and a more rigid structure tend to decrease the energy dissipated by the system. Contrarily, more load on the backstay results in a higher energy dissipation which might be explained by more flapping of the sails because of a looser leech, despite their reduced camber. In both cases, the useful power predicted by the simulation is higher for a looser rig, corresponding to more cambered sails. Direct application of this conclusion (looser rig/fuller sails resulting in a higher driving force) to the real case must be moderated by the 
assumption of inviscid flow used in this work which is known to lead to an optimal sail shape with more camber than the actual optimal because flow separation is not modelled. Moreover, the side force and heeling moment must be considered as well to optimise the sails trim as they affect the performance due to leeway and heel. A full Velocity Prediction Program including hydrodynamic forces must be used for a realistic optimisation.

\section{Acknowledgements}

The authors are grateful to K-Epsilon company for continuous collaboration. This work was supported by the French Naval Academy and Brest Metropole Océane.

\section{References}

Abbott I. H., v. D. A. E., 1949. Theory of Wing Sections, including a summary of airfoil data. Dover publications, Inc.

Augier, B., Bot, P., Hauville, F., Durand, M., 2010. Experimental validation of unsteady models for Wind / Sails / Rigging Fluid structure interaction. International Conference on Innovation in High Performance Sailing Yachts, Lorient, France.

Augier, B., Bot, P., Hauville, F., Durand, M., 2011. Experimental full scale study on yacht sails and rig under unsteady sailing conditions and comparison to fluid structure interaction unsteady models. The 20th Chesapeake Sailing Yacht Symposium, Annapolis USA. 
Augier, B., Bot, P., Hauville, F., Durand, M., 2012. Experimental validation of unsteady models for fluid structure interaction: Application to yacht sails and rigs. Journal of Wind Engineering and Industrial Aerodynamics 101 (0), 53 66.

Augier, B., Bot, P., Hauville, F., Durand, M., 2013. Dynamic Behaviour of a Flexible Yacht Sail Plan. Ocean Engineering 66, 32-43.

Chapin, V., Heppel, P., 2010. Performance optimization of interacting sails through fluid structure coupling. 2nd International Conference on Innovation in High Performance Sailing Yachts, Lorient, France.

Charvet, T., Hauville, F., Huberson, S., 1996. Numerical simulation of the flow over sails in real sailing conditions. Journal of Wind Engineering and Industrial Aerodynamics 63 (1-3), $111-129$.

Fitt, A. D., Lattimer, T. R. B., 2000. On the unsteady motion of two-dimentional sails. J. Appl. Maths 65, 147-171.

Flay, R. G. J., 1996. A twisted flow wind tunnel for testing yacht sails. Journal of Wind Engineering and Industrial Aerodynamics 63 (13), 171 - 182, special issue on sail aerodynamics.

Fossati, F., 2010. Aero-Hydrodynamics and the Performance of Sailing Yachts: The Science Behind Sailing Yachts and Their Design. Adlard Coles Nautical.

Fossati, F., Muggiasca, S., 2009. Sails Aerodynamic Behavior in dynamic condition. The 19th Chesapeake Sailing Yacht Symposium, Annapolis, USA. 
Fossati, F., Muggiasca, S., 2010. Numerical modelling of sail aerodynamic behavior in dynamic conditions. 2nd International Conference on Innovation in High Performance Sailing Yachts, Lorient, France.

Fossati, F., Muggiasca, S., 2011. Experimental investigation of sail aerodynamic behavior in dynamic conditions. Journal of sailboat technology (03).

Fossati, F., Muggiasca, S., 2012. An experimental investigation of unsteady sail aerodynamics including sail flexibility. 4th High Performance Yacht Design Conference Auckland, New Zeeland.

Garrett, R., 1996. The symmetry of sailing: the physics of sailing for yachtsmen. Sheridan House, Inc.

Gerhardt, F., Flay, R. G. J., Richards, P. J., 2011. Unsteady aerodynamics of two interacting yacht sails in two-dimensional potential flow. Journal of Fluid Mechanics 668 (1), 551-581.

Glauert, H., 1926. The Elements of Aerofoil and Airscrew Theory. Cambridge University Press.

Hauville, F., Durand, M., Roux, Y., 2008. Aero elastic model applied to the deformation of a rig. European Journal of Environmental and Civil Engineering $12(5), 549-560$.

Jackson, P., 2001. An improved upwind sail model for vpps. The 15th Chesapeake Sailing Yacht Symposium, Annapolis, USA.

Keuning, J., Vermeulen, K., de Ridder, E., 2005. A generic mathematical model 
for the manoeuvring and tacking of a sailing yacht. The 17th Chesapeake Sailing Yacht Symposium, Annapolis, USA, 143-163.

Marchaj, C., 1996. Sail performance: techniques to maximize sail power. International Marine/Ragged Mountain Press.

Masuyama, Y., Fukasawa, T., 1997. Full scale measurement of sail force and the validation of numerical calculation method. The 13th Chesapeake Sailing Yacht Symposium, Annapolis, USA.

Masuyama, Y., Tahara, Y., Fukasawa, T., Maeda, N., 1993. Dynamic performance of sailing cruiser by a full scale sea reality. The 11th Chesapeake Sailing Yacht Symposium, Annapolis, USA.

Renzsh, H., Graf, K., 2010. Fluid Structure Interaction simulation of spinnakers - Getting closer to reality. 2nd International Conference on Innovation in High Performance Sailing Yachts, Lorient, France.

Richardt, T., Harries, S., Hochkirch, K., 2005. Maneuvering simulations for ships and sailing yachts using friendship-equilibrium as an open modular workbench. International Euro-Conference on Computer Applications and Information Technology in the Maritime Industries.

Roux, Y., Durand, M., Leroyer, A., Queutey, P., Visonneau, M., Raymond, J., Finot, J., Hauville, F., Purwanto, A., 2008. Strongly coupled VPP and CFD RANSE code for sailing yacht performance prediction. 3rd High Performance Yacht Design Conference Auckland, New Zeeland.

Roux, Y., Huberson, S., Hauville, F., Boin, J., Guilbaud, M., Ba, M., 2002. Yacht 
performance prediction: Towards a numerical vpp. High Performance Yacht Design Conference.

Schoop, H., Bessert, N., 2001. Instationary aeroelastic computation of yacht sails. International Journal for Numerical Methods in Engineering 52 (8), 787-803. 